\title{
Design and Finite Element Analysis of Water Jet Energy Accumulator Barrel
}

\author{
Zhang Yuxian, Yang mengke, Wang Hong , Liu Binbin \\ Guilin University of Electronic Technology, Guilin 541004, China
}

\begin{abstract}
In order to solve the yielding failure problem of water jet energy accumulator barrel due to high pressure, the prestressed composite structure design and the theory of equal strength are used to determine the parameters of accumulator barrel, such as the internal cylinder of the composite cylinder, the radii of the inner and outer cylinders, the radius of sub-layer and the optimal interference. Then the ANSYS software is used to analyze the cylinder model with the finite element method, the equivalent stress of the effective node along the wall thickness of the simulation model is obtained by path extraction method and the distribution curves of the equivalent stress along the wall thickness with different internal pressure are also obtained. By analyzing the simulated results and curves, the design parameters of accumulator barrel meet the expected strength requirement.
\end{abstract}

\section{Introduction}

Water jet cutting is a kind of non-conventional machining method, which uses high speed and high kinetic energy fluid medium to working. The cutting process belongs to cold cutting, which does not affect the material structure, and has no phenomenon of mechanical stress deformation and warpage. Therefore, which is widely used in aviation, military industry, electronics and other fields.

Accumulator is an important auxiliary component of high pressure water in water jet cutting. It can save pressure, absorb shock and eliminate pulsation. The carrying capacity directly influences the cutting performance. In order to improve the actual strength of the barrel, in this paper, accumulator in the use of the process often due to high pressure and yield failure problem, the design of prestressed composite structure of accumulator cylinder, and make use of finite element method to valify, in order to improve the bearing capacity of the accumulator.

\section{The failure form of accumulator barrel}

Taking a company's WL-01025A water cut pressurization system (see Figure 1) as an example, to explain the function of the accumulator. After pressing the lowpressure water to the compound supercharger, it passes through the pipeline and enters the accumulator from the accumulator plug head. At this time, the accumulator barrel is subjected to approximate $400 \mathrm{mpa}$, it also absorbs shock and eliminates pulsation [1].

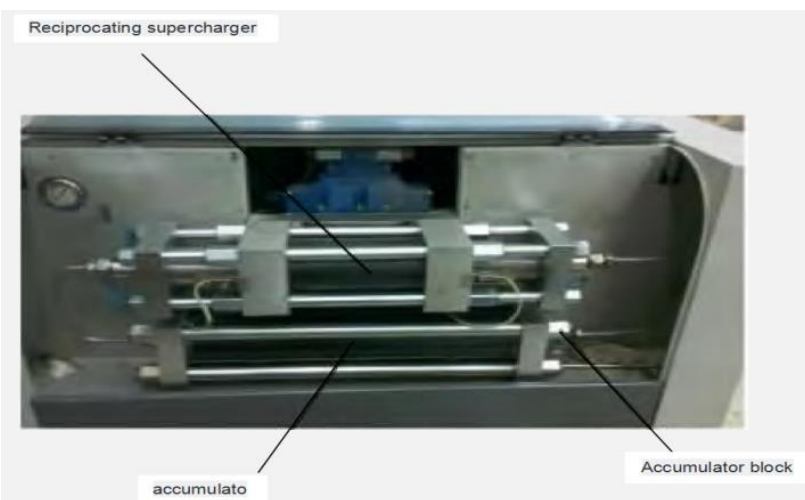

Figure 1. WL-01025A type water cutting supercharging system drawing

In the actual working process, the accumulator tube may have yield deformation, to make the plug seal failure, which resulting in high pressure water leakage, and water reducing rapidly in energy storage, it can't achieve the purpose of cutting [2]. The design of accumulator cylinder, using elastic failure criterion. When the cylinder maximum stress reaches the yield limit, the accumulator cylinder bring about yield failure.

\section{Structure design of accumulator barrel}

\subsection{Problems existing in Single layer thick walled cylinder}

If water cutting accumulator in the design of single layer thick wall cylinder, under high load, the stress distribution is extremely inhomogeneous, according to the fourth strength theory, when the equivalent $s$ 
equal to the yield limit of material, $\sigma_{\mathrm{eq}}=\sigma_{\mathrm{s}}$.accumulator cylinder start to yield, then the diameter ratio $\mathrm{K}=$ $\sqrt{\frac{0.577 \sigma_{s}}{0.577 \sigma_{s}-p_{i}}}$

,thus when internal pressure $p_{i=0.577} \sigma_{s}$, the diameter ratio is close to infinity, when the thick wall cylinder is yielding. From the above analysis, it is limited to increase the load-carrying capacity of the cylinder by increasing the thickness of the wall and using the high strength material. Therefore, it's necessary to change the structure of accumulator barre.

The prestress method can effectively improve the stress distribution of the cylinder and enhance the carrying capacity of the cylinder, in addition, it is simple and effective. In order to excavate the material potential further, the equal strength theory is adopted to design the prestressed cylinder.

\subsection{Equal strength design of prestressed composite cylinder}

\subsubsection{Based on the equal strength theory of the cylinder diameter parameters}

The relative structural parameters of the accumulator's prestressed composite cylinder are shown in figure 2 . Figure $2: r_{a}$ is inner barrel radius of the inner wall; $r_{b}$ is layered radius; $r_{c}$ is inner wall radius of the outer cylinder; $\delta 1$ is inner cylinder radial shrinkage; $\delta 2$ is cylinder radial expansion tensor; $\delta$ max is the optimal interference of the inner cylinder which is relative to the outer cylinder; $p_{1,2}$ for shrunk interface pressure.

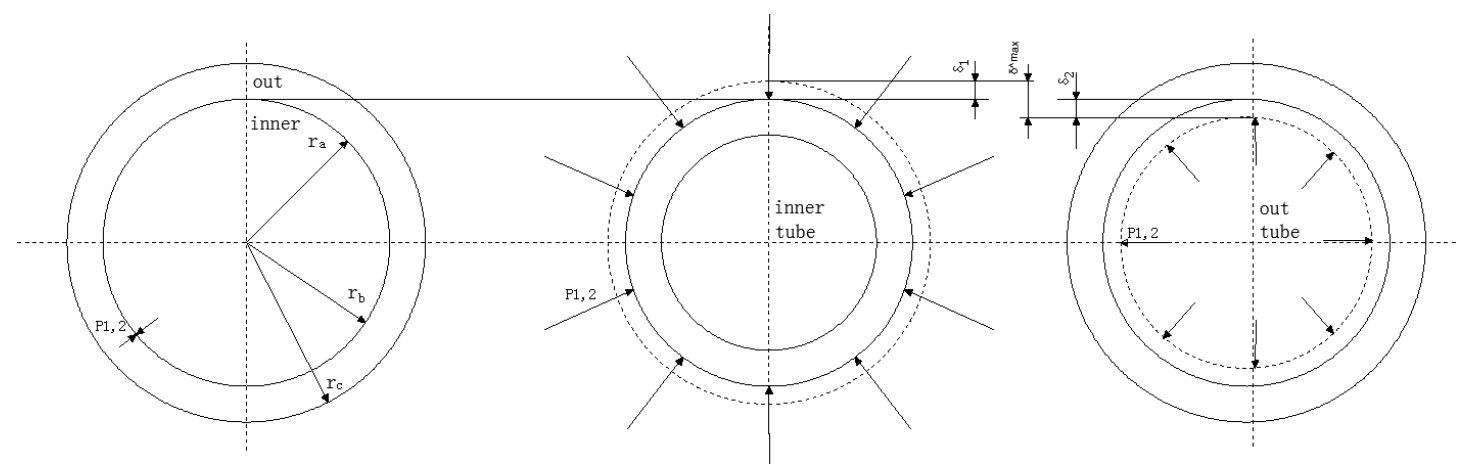

Figure 2. Structural parameters of prestressed composite cylinder sketch map

According to the elastoplastic theory of the cylinder,when maximum shear stress, when maximum shear stress is $\tau_{\max }=\frac{\sigma_{s}}{\sqrt{3}}$ ,the maximum permissible internal pressure $p_{i}$ is

$$
p_{i}=\frac{2 \sigma_{s}}{\sqrt{3}}\left[1-\frac{1}{2}\left(\frac{r_{b}^{2}}{r_{c}^{2}}-\frac{r_{a}^{2}}{r_{b}^{2}}\right)\right]
$$

According to the equivalent strength design theory, it can be available[3].

$$
p_{i}=\frac{2 \sigma_{s}}{\sqrt{3}}\left[1-\frac{1}{K}\right]
$$

\subsection{2 optimum interference calculation}

Timoshenko deduction interface pressure formula of cylinder is

$$
p_{1,2}=\frac{\delta E}{2 r_{b}} \frac{\left(K^{2}-K_{1}^{2}\right)\left(K_{1}^{2}-1\right)}{K_{1}^{2}(K-1)}
$$

The optimum interference amount of the inner cylinder relative to the outer cylinder can be obtained[4] $\delta_{\max }$

$$
\delta_{\max }=\frac{2 \sigma_{s}}{\sqrt{3} E}\left(\frac{\sqrt{r_{a} r_{c}}\left(r_{c}-r_{a}\right)}{r_{c}}\right)=\frac{2 r_{a} \sigma_{s}}{\sqrt{3 K} E}(K-1)=\delta_{1}+\delta_{2}
$$

\section{3 instance calculation}

A cylinder, radius of inner wall of cylinder $r_{a}=23 \mathrm{~mm}$, maximum allowable internal pressure $p=500 M P a$. Both inner and outer cylinder materials are selected $0 \mathrm{Cr} 17 \mathrm{Ni} 4 \mathrm{CuNb}$, modulus of elasticity $E=213 \mathrm{GPa}$,yield strength $\sigma_{s}=856 \mathrm{MPa}$,Poisson ratio $\mu=0.27$,tangential modulu $E_{T}=210 \mathrm{MPa}$.

The concrete parameters of prestressed composite cylinder are as follows:inner radius of inner barrel $^{r_{a}=23.0 \mathrm{~mm}}$,delamination radius ${ }_{b}=32.7 \mathrm{~mm}$,outer radius 
of outer cylinder $\quad r_{c}=46.5 \mathrm{~mm} \quad$,optimum interference $\delta_{\max }=0.0768 \mathrm{~mm}$.

\section{Finite element analysis of accumulator prestressed cylinder}

\subsection{The establishment of finite element model}

Since the diameter of the accumulator is larger than the length and the material is nonlinear, the analysis belongs to the problem of plane strain and contact nonlinearity.

In addition, the model is symmetric and can be simplified to the two-dimensional $1 / 4$ model.According to the foregoing to establish model,the selection can be approximated by simulation with elastoplastic properties,two dimensional plane element of large deformation and large strain cylinder materials PLANE183, and Setting material mechanical parameters, the bilinear kinematic hardening model (BKIN) is adopted to simulate the material nonlinearity at the same time[5].

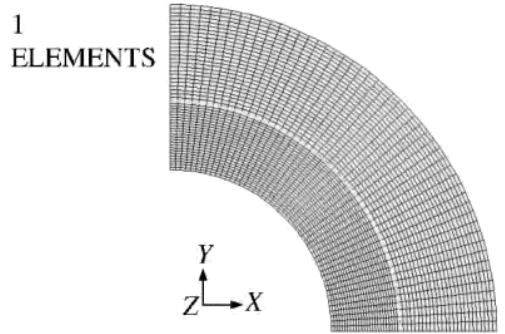

Figure 3. dividing mesh of prestressed cylinder model

As shown in Figure 3, the cell shape is set to a quadrilateral,the mesh is mapped by mapping,set the contact to simulate the external wall of the inner cylinder ectotheca and the outer cylinder wall,as to get preload effect. in order to obtain better convergence, the contact stiffness of the penalty function is set to 0.1 ; all nodes which $\mathrm{X}=0$ and $\mathrm{Y}=0$, applying 0 degrees of freedom for $\mathrm{X}, \mathrm{Y}$ direction respectively . The entire loading process is set from 0 to $50 \mathrm{MPa}$ to $600 \mathrm{MPa}$, and the solution process is divided into 13 load steps[6].

\subsection{Finite element post processing and stress data analysis}

The different load step equivalent stress nephogram is studied, When the internal pressure of the cylinder is less than $500 \mathrm{MPa}$, the equivalent stress in the cylinder is no more than the yield limit of the cylinder material,which the whole cylinder in the elastic stage does not yield; when the internal pressure is $500 \mathrm{MPa}$, the largest the value of equivalent stress of the cylinder wall and the inner wall of the barrel, and the outer tube, and the maximum equivalent stress is $856 \mathrm{MPa}$, just to reach the material yield limit, that under $500 \mathrm{MPa}$ pressure, the inner cylinder wall and the inner wall of the outer cylinder to yield( After $1 / 4$ expansion, the $500 \mathrm{MPa}$ stress distribution nephogram is shown in Figure 4); when the internal pressure is greater than $500 \mathrm{MPa}$, the inner wall of the cylinder inner wall and outer cylinder appear yield regions (under internal pressure of $600 \mathrm{MPa}$, prestressed cylinder equivalent stress distribution is showed in Figure 5 ).
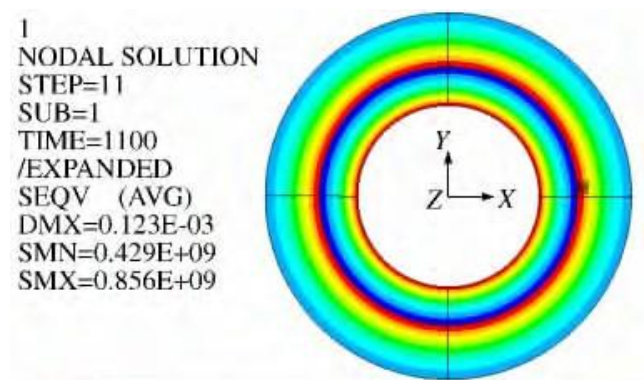

$0.429 \mathrm{E}+09 \quad 0.524 \mathrm{E}+09 \quad 0.619 \mathrm{E}+09 \quad 0.714 \mathrm{E}+09 \quad 0.809 \mathrm{E}+09$ $0.476 \mathrm{E}+09 \quad 0.571 \mathrm{E}+09 \quad 0.666 \mathrm{E}+09 \quad 0.761 \mathrm{E}+09 \quad 0.856 \mathrm{E}+09$ Figure 4. The equivalent stress distribution of $500 \mathrm{MPa}$ prestressed cylinder

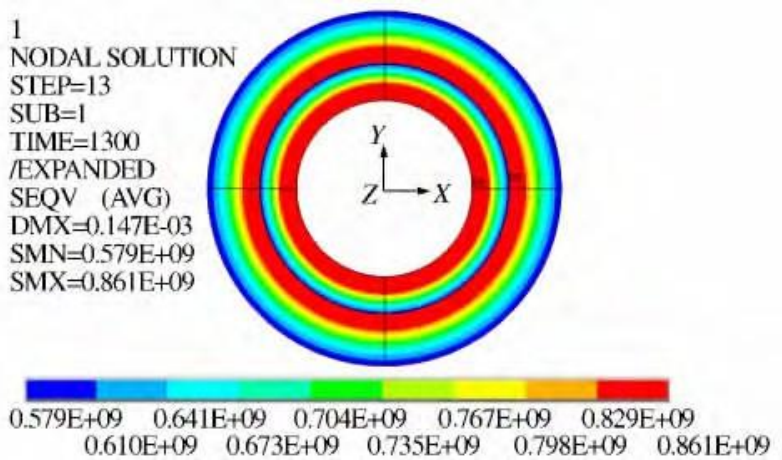

Figure 5. The equivalent stress distribution of $600 \mathrm{MPa}$ prestressed cylinder

In order to further study on the equivalent stress distribution along the wall thickness at $100 \mathrm{MPa}$ to 600 $\mathrm{MPa}$ pressure range, with $100 \mathrm{MPa}$ steps and load in incremental manner, the cylinder continuous discretization compression process,along the wall thickness of the cylinder, the path is set up and the equivalent stress value of the effective node is extracted. The stress distribution curve along the wall thickness under different internal pressure is processed by MATLAB software[7].( See Figure 6)

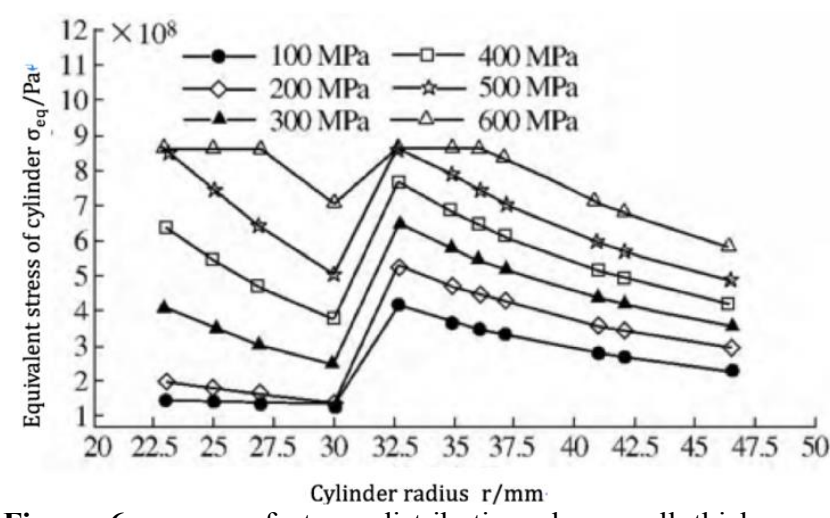

Figure 6. curves of stress distribution along wall thickness under different internal pressures

As can be seen from Figure 6, when the internal pressure is in the range of $100 \mathrm{MPa}$ to $400 \mathrm{MPa}$, the equivalent stress within the cylinder does not reach the yield limit, and the variation tendency in equivalent stress 
along the wall thickness is similarly. The trends performance are: the inner cylinder, the equivalent stress along the wall thickness decreases, the interface to the inner cylinder and the outer cylinder stress increases suddenly, then along the wall thickness decreases until to the outer cylinder; Under internal pressure is $500 \mathrm{MPa}$, and the trend is similar to the before, but yield point along will be showed along the wall thickness near 23 $\mathrm{mm}$ and $33 \mathrm{~mm}$; while the cylinder wall thickness of 33 $\mathrm{mm}$ to $36 \mathrm{~mm}$ the range is also a large yield.

\section{Conclusion}

1) the finite element analysis of the designed accumulator barrel is carried out by using ANSYS software, and the stress results show that the design parameters of the prestressed composite cylinder meet the requirements of the expected strength.

2) From the cylinder under different pressure along the wall thickness equivalent stress distribution curve, it can be seen: when the yield stress along the wall thickness isn't appeared, the stress distribution trend similar; Yielding, yield point first appeared in the inner wall of the inner tube and the inner wall of the outer cylinder, and with the increasing of pressure, the yield region will expand respectively from the inner wall of the inner barrel and the inner wall of the outer cylinder.

3 ) the finite element analysis method can effectively solve the problem of prestressed cylinder touching and nonlinear solution, and can simulate in complex compression environment accurately. It can provide the basis for improving the stress distribution of the extra high pressure cylinder.

\section{References}

1. Yang M G, Yu F, Kang C. et al. Study on moving characteristics of reciprocating intensifier [J] . Drainage and Irrigation Machinery,2009,27 ( 5 ) : 332-336 ( in Chinese)

2. Guo Z F, Qin J P, Zheng Z Z. The research on superhigh pressure prestress composite cylinder of the water cutting Jet [J]. Chinese Hydraulics and Pneumatics, 2004( 4) : 7-10 (in Chinese)

3. $\mathrm{Yu} T \mathrm{~T}$, Xue $\mathrm{P}$. Engineering elasticity and plasticity $[\mathrm{M}]$. 2nd ed . Beijing: Higher Education Press, 2010: 123-135( in Chinese)

4. Zhang $\mathrm{Y} \mathrm{X}$, Wang $\mathrm{H}$. The optimal design of shrunk cylinder super high pressure $[\mathrm{J}]$. Machine Design and Research, 2006, 22( 5) : 115-117 ( in Chinese)

5. Xu C J, Li D S, Dou J P,et al. FEA of cylinder body for ultra-high pressure \& shrink sleeve vessel [J]. China Metal forming Equipment \& Manufacturing Technology, 2011, (6) : 83-86 ( in Chinese)

6. Xiao Sa Studio, Zhang S Q. The new and classic course about ANSYS and workbench [M] . Beijing: Electronic Industry Press, 2004: $178-180$ ( in Chinese)

7. Wang $\mathrm{Z}$ L, Gong $\mathrm{C}$, He Q. Master science computer of MATLAB [M]. Beijing: Electronic Industry Press, 2009: 221-239 (in Chinese) 